\title{
QSO absorption lines, winds, and the high-redshift intergalactic medium
}

\author{
Robert F. Carswell \\ Institute of Astronomy, Madingley Road, Cambridge CB30HA, UK
}

\begin{abstract}
The close similarities in the spectra of the Ly $\alpha$ absorption forests in separate images of gravitationally lensed QSOs at high redshifts provide upper limits for the filling factor of the universe for gas that has undergone recent hydrodynamic disturbances. Strong winds blowing for a substantial fraction of the local Hubble time do not significantly affect the low density regions which fill a large fraction of the volume of the universe. Many of the higher density absorbers appear to be photo-ionized and show some heavy element enrichment. They also contain a cosmologically significant fraction of the baryons and the heavy elements in the universe at that stage of its evolution.
\end{abstract}

\section{Introduction}

The absorption lines seen in the spectra of high redshift quasars provide a powerful probe into the conditions in the gaseous component of the high redshift universe. Large telescopes provide high signal-to-noise ratio spectra with resolution $R \simeq 50000$ or more, so the thermal line widths in gas with temperature $T \simeq 10^{4} \mathrm{~K}$ may be seen. Also, computer simulations of the growth of structure in the universe provide the material for detailed comparisons between the models and the data at an unprecedented level. Studies of the structure of the intergalactic medium (abbreviated to IGM generally) at high redshifts tend to concentrate on the 'Ly $\alpha$ forest' - the ubiquitous absorption by Ly $\alpha$ which fills the spectra of QSOs all the way up to their emission redshifts. The review by Rauch (1998) shows what had been achieved four years ago, and subsequent developments have reinforced and refined the general agreement between the model results and the data.

Among the more recent developments is the galaxy-intergalactic medium interaction. How the formation of galaxies, and their star formation and recycling of processed material into the intergalactic medium affects the properties of the medium. This has followed the work of Songaila \& Cowie (1996), who showed that the denser regions of the IGM have a significant heavy element content, and so overturned the notion which had previously been held that the intergalactic medium predominantly contained gas which had relative abundances which came from primordial nucleosynthesis. The most likely source of the heavy element enrichment is materal ejected from galaxies or protogalaxies at some stage. There are several possibilities - during Population III star formation at very early stages, through disruption of small 'protogalaxies' at redshifts $5 \lesssim z \lesssim 20$, or ejecta from massive galaxies at redshifts $z \lesssim 8$ or so (Aguirre et 


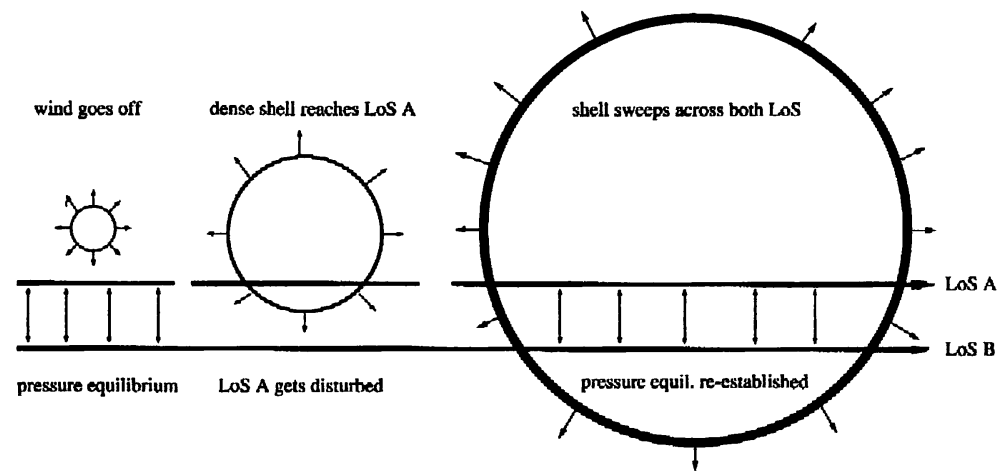

spectrum A

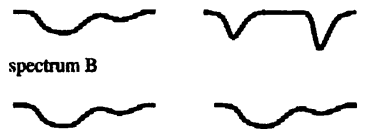

if sound crossing time < shell crossing time:

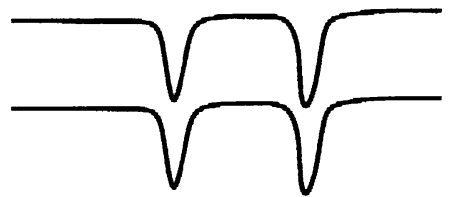

Figure 1. Left: A wind starts near two lines of sight, A and B. The spectra are identical until (middle) the expanding shell hits the first sightline. Later, (right) after the shell has slowed sufficiently, equilibrium is re-established between the two sightlines, and the absorption pattern is similar in the two again, though different from the initial state.

al. 2001 and references therein). Whichever turns out to be the dominant mechanism, the role of galactic winds at high redshift is important. The signatures, and consequences, of galactic winds are considered further in the following sections.

\section{Galactic winds}

Winds and superwinds from galaxies, notably starburst galaxies, are regarded as the only plausible source for substantial heavy element enrichment of the intergalactic medium. Heckman et al. (2000), from an extensive study of 32 objects, show that local starburst galaxies have winds with velocities of $400-600 \mathrm{~km} \mathrm{~s}^{-1}$ and inferred mass loss rates of order $10-100 \mathrm{M}_{\odot}$ per year, comparable with the star formation rates. At high redshifts, where starburst galaxies are frequently found, similar behaviour is seen (Steidel 2001; Pettini in these Proceedings) even up to redshift $z=5.2$ (Dawson et al. 2002).

We can infer something about the wind timescales and filling factors by considering their effect on the intergalactic medium. This involves comparing two regions of the IGM to look for differences arising from the galactic winds. Rauch et al. (2001) have done this for two images of the gravitationally lensed QSO $1422+231$. They find that the Ly $\alpha$ forest lines in the two sightlines are almost identical, and so the rms fluctuations in the (log) baryon density field are $\varsigma 3 \times 10^{-2}$ on a scale of $\sim 100 \mathrm{pc}$. From the fraction of absorption lines which could be different on this lengthscale, they derive an upper limit of $20 \%$ for the volume filling factor of the universe by strong winds, which blow for a substantial 
$H E 2217-2818 \quad z=2.181155$

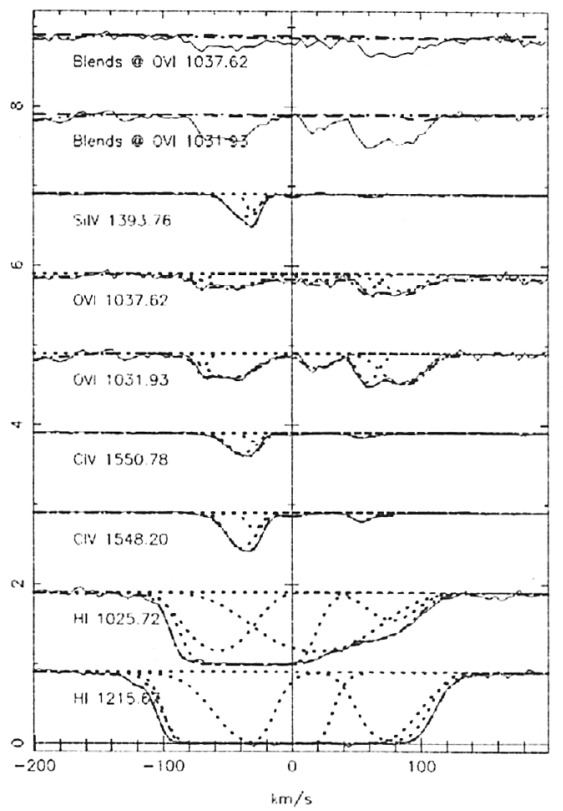

HE $1122-i 648 z=2.080162$

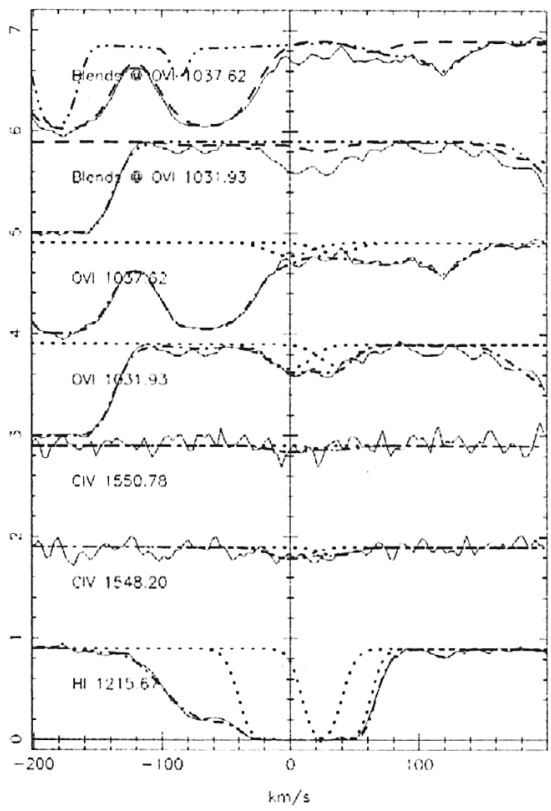

Figure 2. The redshift systems at $z=2.180$ towards HE 2217-2818 where the O VI and CIV structure do not match well, and $z=2.080$ towards HE 1122 1648 where the $\mathrm{CIV}$ and $\mathrm{O}$ VI redshifts agree. Both are on a velocity scale centered on the redshift given above each figure. Total profile fits to the data are shown as dot-dashed lines. The CIV region in the right-hand panel has been re-scaled by a factor 5 , so the zero level is 4.5 units below the continuum. Individual components to the fit are shown as dotted lines. The upper two spectra in each case show the O VI line regions with the blended Ly $\alpha$ lines shown as dot-dot-dot-dash, and other well-constrained blends as dashed lines.

fraction of the Hubble time (at $z=3.3$ ). The scenario here is best described by figure 10 from Rauch et al. (2001). Winds which originate close to the epoch of observation would not be detected, because their volume filling factor is small, and old weak winds would be missed because of their limited lifetime.

\section{IGM enrichment}

C IV is generally present in Ly $\alpha$ forest absorption systems with neutral hydrogen column densites $N_{\mathrm{HI}} \gtrsim 10^{14.5} \mathrm{~cm}^{-2}$ (Songaila \& Cowie 1996; Boksenberg 1997), i.e., in regions of the universe where, at $z \simeq 3$, the densities are $\rho / \bar{\rho} \gtrsim 5$ at that epoch. Data pixel search techniques, where the $\mathrm{O}$ VI and H I data corresponding to a given $\mathrm{HI}$ optical depth is binned, suggest that it is also present at lower densities (Cowie \& Songaila 1998).

A major consideration in choosing $\mathrm{C}$ IV is that the rest wavelengths of the C IV doublet (1548.20 and $1550.78 \AA$ ) are greater than Ly $\alpha(1215.67 \AA)$, so over a reasonable redshift range there is no confusion with Lyman forest lines. However, $\mathrm{O}$ VI is expected to be considerably more sensitive than CIV for lower density 
regions (Chaffee et al. 1986; Rauch, Haehnelt \& Steinmetz 1997). The difficulty with O VI is that the absorption lines, at 1031.93 and $1037.62 \AA$, are confused by Ly $\alpha$ and higher order Lyman lines in the Ly $\alpha$ forest. This makes them very difficult to find at redshifts $\gtrsim 3$ where the Lyman line density is high, but at lower redshifts the Ly $\alpha$ forest is less dense so O VI could be detected. Pixel searches (Schaye et al. 2000) show that O VI is present at these redshifts, and two recent studies by Simcoe (2001), Simcoe et al. (2002) and Carswell et al. (2002) give analyses based on $\mathrm{O}$ VI detections in a number of redshift systems.

While the data on individual O vi systems are still a bit sparse, there are some interesting tentative results. Both the Simcoe et al. (2002) and Carswell et al. (2002) investigations show that C IV is also present in many cases, and that the $\mathrm{HI}$ column density is usually $\gtrsim 10^{14.5} \mathrm{~cm}^{-2}$. Simcoe et al. find that O VI absorbers are associated with complex systems and often other lower ionization species are present, and that the detailed velocity structure does not generally match that of the other heavy elements. Carswell et al. on the other hand, find several cases where the O VI and CIV velocity structure match each other. Examples of each type (from Carswell et al. ) are shown in Figure 2.

The reason for the difference may be that the Simcoe et al. (2002) systems generally have a neutral hydrogen column densities $N_{\mathrm{HI}}>10^{15} \mathrm{~cm}^{-2}$ or more, while the ones found by Carswell et al. (2002) generally have $N_{\mathrm{HI}}$ less than that. Photo-ionization modeling allows density estimates to be obtained. The higher column density complexes are associated with densities $\rho / \bar{\rho} \gtrsim 100$, while the lower column density ones have $\rho / \bar{\rho} \approx 10-20$, and heavy element abundances, typically $10^{-2}-10^{-2.5}$ solar. The higher density systems are associated with condensations, so it is perhaps not surprising that they show complex structure. The lower densities here correspond to filaments (see Rauch 1998), and the photo-ionization model densities are in good agreement with those inferred from the H I column density for general hydrodynamic models (Schaye 2001). Thus it appears that these systems have been metal-enriched without the enrichment process having seriously perturbed them.

It is possible that the assumption of photo-ionization is incorrect, of course, and that collisional ionization is important. This requires temperatures of order $230000 \mathrm{~K}$, and so $\mathrm{HI}$ line widths much greater than is seen. A way around this is for the Lyman lines arising in separate physical regions, but then the $\mathrm{H}$ I column densities associated with the O VI and C IV would be lower. Then in all cases a high metallicity collisionally-ionized region would be associated in some way with a relatively cool low abundance one.

Aguirre et al. (2001) have estimated the median metallicity vs. overdensity at redshift $z=3$ for a galactic wind model, and find that metallicities $\sim 10^{-2}$ solar or more are likely for $\rho / \bar{\rho} \gtrsim 10$, and close to zero for lower densities. The redshifts for the observed cases described above are lower, so the possible propagation times longer and enrichment to lower density regions may occur. The O VI measurements are not sensitive enough in any case to test for possible abundance inhomogeneities at the expected level.

\section{High abundance regions}

While almost all systems show heavy element enrichment at about the expected level, there are some anomalies which show considerably higher heavy element 


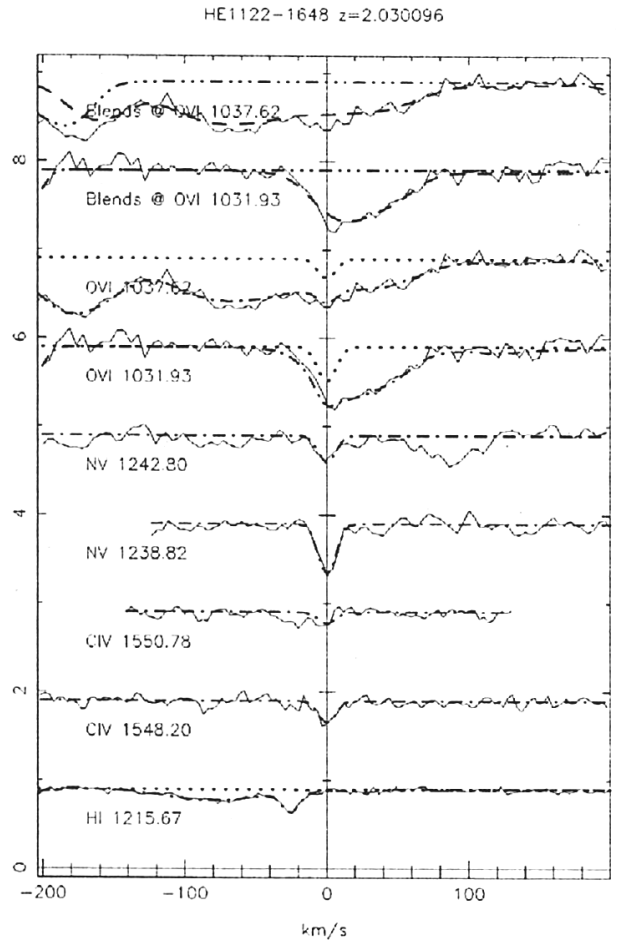

Figure 3. The redshift system $z=2.030$ towards HE 1122-1648 showing the $\mathrm{H} \mathrm{I}, \mathrm{CIV}, \mathrm{NV}$ and $\mathrm{OVI}$ absorption lines. The $\mathrm{CIV}$ and $\mathrm{N} \mathrm{V}$ regions have been scaled by a factor three. Other details as for Figure 2.

abundances. The most striking is at $z=2.030$ towards HE 1122-1648, where $\mathrm{CIV}, \mathrm{NV}$ and OVI are seen, and Ly $\alpha$ is remarkable for its absence. The line profiles are shown in Figure 3.

The hydrogen density inferred from photionization models based on the $\mathrm{C}$ IV/O vi ratio is $\sim 10^{-4} \mathrm{~cm}^{-2}$, and the weak Ly $\alpha$ then implies that $\mathrm{C} / \mathrm{H}$ is $5 \times$ solar. If, instead, collision ionization is the dominant mechanism, then $\mathrm{C} / \mathrm{H}$ is $\sim 1.5 \times$ solar. The presence of $\mathrm{N} \mathrm{V}$ is unusual at these redshifts, and suggests that secondary nitrogen production may be important for this system. Indeed, the models suggest that $\mathrm{N} / \mathrm{O}$ is about $1-2 \times$ solar. If photo-ionization is important, then the $\mathrm{HI} / \mathrm{H}$ ratio is $10^{-4.6}$, so the size scale for the cloud is small, $\sim 10^{2} \mathrm{pc}$. For the collisional ionization case there is no direct constraint on the cloud density, so a size estimate is not possible. High abundance weak systems are known at lower redshifts (e.g., weak Mg II systems studied by Rigby et al. 2002), and at $z \simeq 2$ they are present in a few objects. The nature of these apparently isolated small systems is a topic for further investigation.

\section{Conclusions}

Galactic winds from starburst galaxies at high redshift are likely to play a significant role in the metal enrichment of the intergalactic medium. Strong winds 
which last for a significant fraction of the age of the Universe at redshifts $z \simeq 3.3$ would have been detected if their volume filling factor exceeded $\sim 20 \%$, but they have not been seen. The detection of heavy elements, particularly C IV and O VI, in overdense regions in the intergalactic medium shows that a significant fraction of the mass (but not necessarily the volume) has been enriched by material which has undergone nuclar processing in stars. The heavy element abundances in many ofthe systems where the heavy elements are found are $\sim 10^{-2}-10^{-2.5}$ solar, consistent with model predictions. There are some, apparently isolated, small high ionization systems with heavy element abundances close to solar.

Acknowledgments. My thanks to Tae-Sun Kim, Michael Rauch, Joop Schaye and Rob Simcoe for discussions, and Rob Simcoe and collaborators for providing me with a copy of their paper in advance of publication.

\section{References}

Aguirre, A., Hernquist, L., Schaye, J., Weinberg, D. H., Katz, N., Gardner, J. 2001, ApJ 560, 599

Boksenberg, A., 1997, in: P. Petitjean \& S. Charlot (eds.), Structure and Evolution of the Intergalactic Medium from QSO Absorption Lines (Paris: Editions Frontières), p. 85

Carswell, R.F., Schaye, J., Kim, T.-S. 2002, ApJ 578, 43

Chaffee, F.H., Foltz, C.B., Bechtold, J., Weymann, R.J. 1986, ApJ 301, 116

Cowie, L.L., Songaila, A. 1998, Nature 394, 44

Dawson, S., Spinrad, H., Stern, D., Dey, A., van Breugel, W., de Vries, W., Reuland, M. 2002, ApJ 570, 92

Heckman, T.M., Lehnert, M.D., Strickland, D.K., Armus, L. 2000, ApJS 129, 493

Rauch, M. 1998, Ann. Review Astron. Astrophys. 36, 267

Rauch, M., Haehnelt, M. G., Steinmetz, M. 1997, ApJ 401, 601

Rauch, M., Sargent, W.L.W., Barlow, T.A., Carswell, R.F. 2001, ApJ 562, 76

Rigby, J.R., Charlton, J.C., Churchill, C.W. 2002, ApJ 565, 743.

Schaye, J., Rauch, M., Sargent, W.L.W., Kim, T.-S. 2000, ApJ (Letters) 541, L1

Schaye, J. 2001, ApJ 559, 507

Simcoe, R.A. 2001, BAAS 198, 26.05

Simcoe, R.A., Sargent, W.L.W., Rauch, M. 2002, ApJ 578, 737

Songaila, A., Cowie, L.L. 1996, AJ 112, 335

Steidel, C.C. 2001, BAAS 198, 52.08

\section{Discussion}

SCHAERER: What are the prospects of determining relative $\mathrm{O} / \mathrm{C}$ abundances in the IGM? This would be very interesting, e.g., to probe if pair creation SNe exist in Pop III (metal-free) stellar populations, as strongly enhanced $\mathrm{O} / \mathrm{C}$ would be expected from such objects ( $c f$. Schaerer 2002, A\&A 382, 28).

CARSwEll: Unfortunately very poor. Really, we need to know ionizing flux shape, and then intensity from an ion ratio, and C VI, O VI, all that is generally seen. C III / $\mathrm{CIV}$ would help, but would still need shape. 